\title{
A Case of Alzheimer's Disease Was Kept Relative Stable with Sequential Therapy for Eight Years
}

\author{
Mingqing Wei ${ }^{*}$, Xuekai Zhangi* ${ }^{*}$, Jing Shi ${ }^{*}$, Jingnian $\mathrm{Ni}^{1}$, Ting $\mathrm{Li}^{1}$, Tao $\mathrm{Lu}^{2}$, Yuanyuan Shi², \\ Liping Zhang ${ }^{3}$, Pengwen Wang', Shenghua Kang1, Fuyun Ma1 , Yumeng Li ${ }^{1}$, \\ Chenmeng Li' Jinzhou Tian" ${ }^{1 \#}$
}

\begin{abstract}
${ }^{1}$ BUCM Neurodegenerative Diseases Center, Dongzhimen Hospital, Beijing University of Chinese Medicine, Beijing, China ${ }^{2}$ School of Life Science, Beijing University of Chinese Medicine, Beijing, China

${ }^{3}$ Department of Radiology, Dongzhimen Hospital, Beijing University of Chinese Medicine, Beijing, China

${ }^{4}$ Key Laboratory of Pharmacology of Chinese Materia Medica, Dongzhimen Hospital, Beijing University of Chinese Medicine, Beijing, China

Email: mingqingwei001@126.com, zhangxuekai2008@live.cn, shijing87@hotmail.com, jingnian_ni@hotmail.com, bjliting@yeah.net, tao_lu@hms.harvard.edu, yshi@LRRI.org, pw_wang@163.com, Lionksh@126.com, fuyunma@163.com,

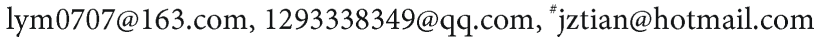

How to cite this paper: Wei, M.Q., Zhang, X.K., Shi, J., Ni, J.N., Li, T., Lu, T., Shi, Y.Y., Zhang, L.P., Wang, P.W., Kang, S.H., Ma, F.Y., Li, Y.M., Li, C.M. and Tian, J.Z. (2017) A Case of Alzheimer's Disease Was Kept Relative Stable with Sequential Therapy for Eight Years. World Journal of Neuroscience, 7, 209-215. https://doi.org/10.4236/wjns.2017.72016

Received: March 1, 2017

Accepted: April 4, 2017

Published: April 7, 2017

Copyright (c) 2017 by authors and Scientific Research Publishing Inc. This work is licensed under the Creative Commons Attribution International License (CC BY 4.0).

http://creativecommons.org/licenses/by/4.0/ (c) (i) Open Access

\begin{abstract}
Background: Although Alzheimer's disease (AD) has been intensively investigated for many years, the effective treatments are largely missing. Commonly used conventional therapy, such as cholinesterase inhibitors (ChEI) and $\mathrm{N}$-methyl $\mathrm{D}$-asparate receptor antagonist, have been generally considered as having symptom-relieving rather than disease-modifying effects. Thus, how to improve cognitive function beyond such effect $\&$ time limitations has become a serious challenge. Aim: In order to solve this challenge, a sequential therapy with the integration of conventional therapy and herbal therapy was applied to $\mathrm{AD}$ patients. Careful clinical observation was conducted in our outpatient setting. Case Presentation: A case of probable $\mathrm{AD}$ received the sequential therapy has achieved relative stable cognition and overall status in eight years. Conclusion: During the treatment of this $\mathrm{AD}$ case in eight years, sequential therapy showed great potential in stabilizing and improving cognition and overall status. Well designed preclinical and clinical studies are needed to investigate the efficacy of sequential therapy for $\mathrm{AD}$ and other type of dementia.
\end{abstract}

\section{Keywords}

Alzheimer's Disease, Sequential Therapy, Cholinesterase Inhibitors, Herbal Medicine 


\section{Introduction}

Alzheimer's disease (AD), the most common cause of dementia in the elderly, will dramatically increase in prevalence during the process of worldwide demographic ageing. It is an irreversible, progressive neurodegenerative disease, which gradually destroys memory, thinking ability, and eventually the ability to carry out the simplest tasks like eating. Memory problems are typically one of the first signs of cognitive impairment related to AD. And non-memory aspects of cognition, such as word-finding, vision/spatial issues, and impaired reasoning or judgment, may also seen in very early stages of $\mathrm{AD}$. With disease progress, Memory loss and confusion get worse, (may have problems recognizing family and friends), executive function also get worse (unable to carry out multistep tasks such as getting dressed, or cope with new situations). In addition, psychiatric symptoms like hallucinations, delusions, and paranoia as well as behavior problems like sleeplessness, wandering, agitation, anxiety, and aggression may occur. At the end stage of the disease, patients cannot communicate and are completely dependent on others for their care, they may be completely bedridden at the end of life. Although AD has been intensively investigated for many years, the effective treatments are largely missing. Commonly used medications, such as cholinesterase inhibitors (ChEI) and N-methyl D-aspartate receptor antagonistara generally considered as symptom-relieving rather than disease-modifying effects [1] [2]. How to improve cognitive function beyond such effects \& time limitations has become a serious challenge. Here we report a case of probable $\mathrm{AD}$ received a sequential therapy, which combined ChEI and herbal medicines, has achieved a significant improvement in eight years of treatment.

\section{Patient Information}

The patient is a retired garment factory worker with primary school education, who was born in 1928. Her first visit of our memory clinic in the company of her daughter on May 25th 2008, complaining about half year of progressive memory loss after a change of living environment (ID 3236151 in our outpatients system). Her daily activities got interfered by her cognition problem. Her neuropsychological assessment in the first visit on Jun-5-2008 revealed that she has slight decrease in global cognition, delayed episodic memory, and calculation/ attention; mild decrease of daily living activities, without obvious depression, anxiety, psychiatric and behavior symptoms. She has related no family history, no relevant sign from physical and neurologic examinations, and no significant abnormality in her laboratory tests. Her ApoE gene test showed a normal E3/E3 genotype. Her MRI scan showed that there were several lacunar infarctions, very mild white matter demyelination around the horns of lateral ventricles, and severe bilateral hippocampus atrophy in coronal FLAIR (Medial temporal lobe atrophy (MTA) scale $=3$ ).

With above information, she was diagnosed as mild dementia due to Alzheimer's disease according to the $\mathrm{AD}$ criteria. She received sequential treatment protocol, which integrated conventional therapy and herbal therapy, that is do- 
nepezil hydrochloride $5 \mathrm{mg}$ once a day and herbal medicine twice a day. Her cognitive function was obviously improved for about 10 months (detailed scores see Figure 1), then gradually falling back to baseline at around 21 months (MMSE change trend see Figure 2). After the relatively stable period with our combined therapy, the patient stopped the treatment for about 40 months. During this time, the patient endured a dramatic drop in memory (MMSE drop to 16). She was brought back to our clinic by her family since September 24, 2013. After three months' combined treatment, her MMSE score improve significantly from 16 to 21 . Interestingly, her memory start to fall after then, nearly back to where she restarted $(\mathrm{MMSE}=17)$ after about six months of treatment. However,

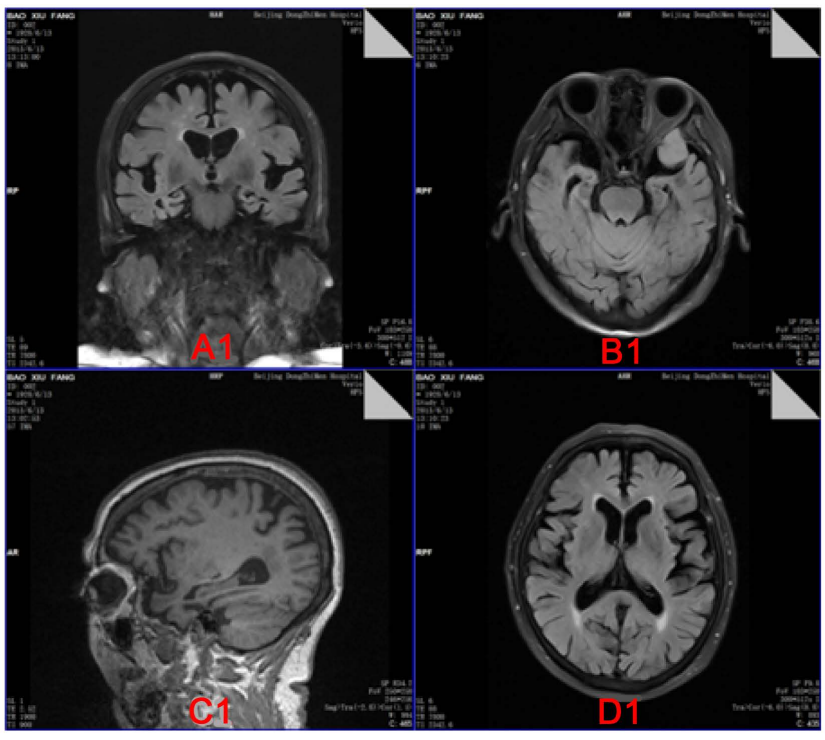

Cranial MRI scan of June 13, 2013

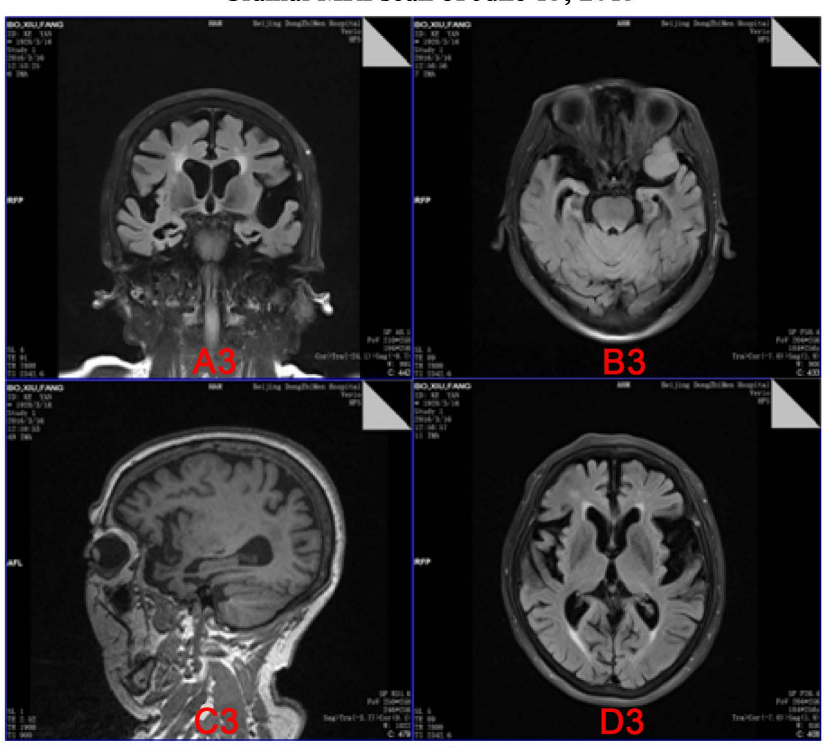

Cranial MRI scan of March 16, 2016

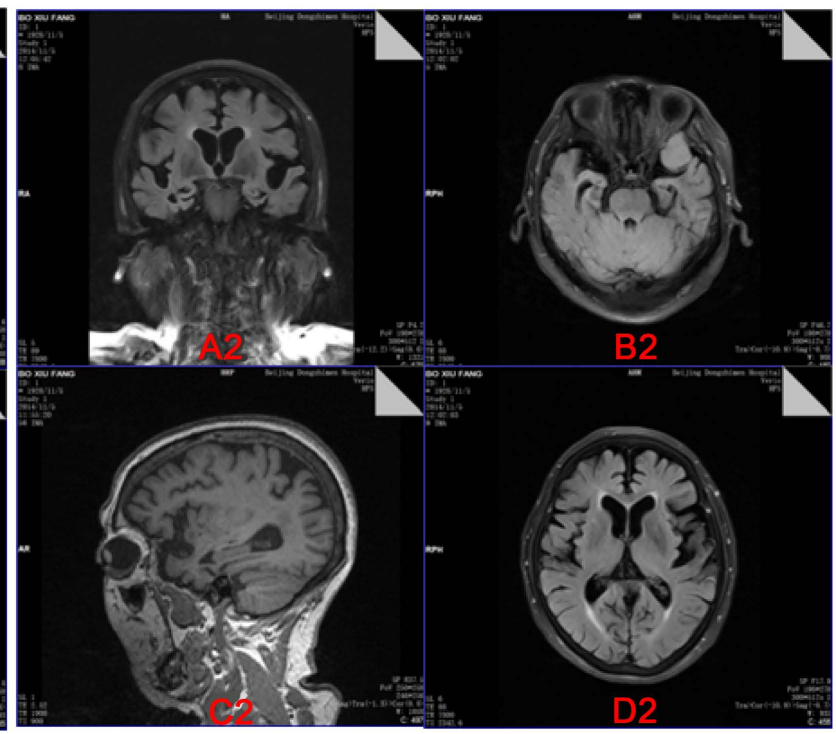

Cranial MRI scan of November 5, 2014

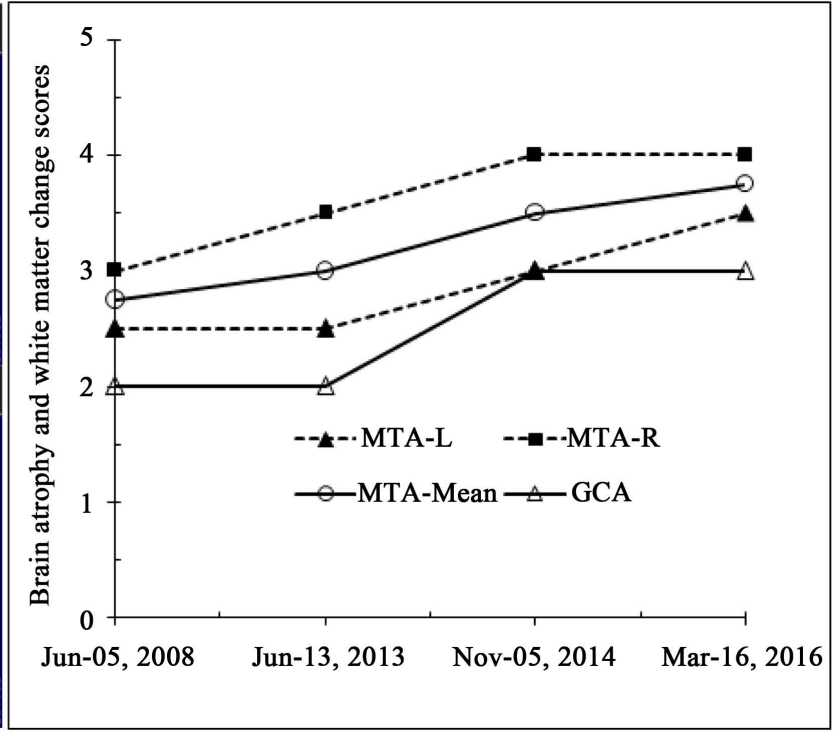

Brain atrophy and white matter change scores of cranial MRI scan

Figure 1. Brain atrophy and white matter changes of cranial MRI scan. Note: (A), Coronal FLAIR, MTA scale was assessed; (B) Axial FLAIR; (C) Sagittal T1 image, GCA scales was assessed; (D) Axial FLAIR. There was obvious increase of MTA scale and GCA scale, from June 5, 2008 to March 16, 2016. However, there are no significant aggravation of the white matter demyelination and lacunar infarction. 


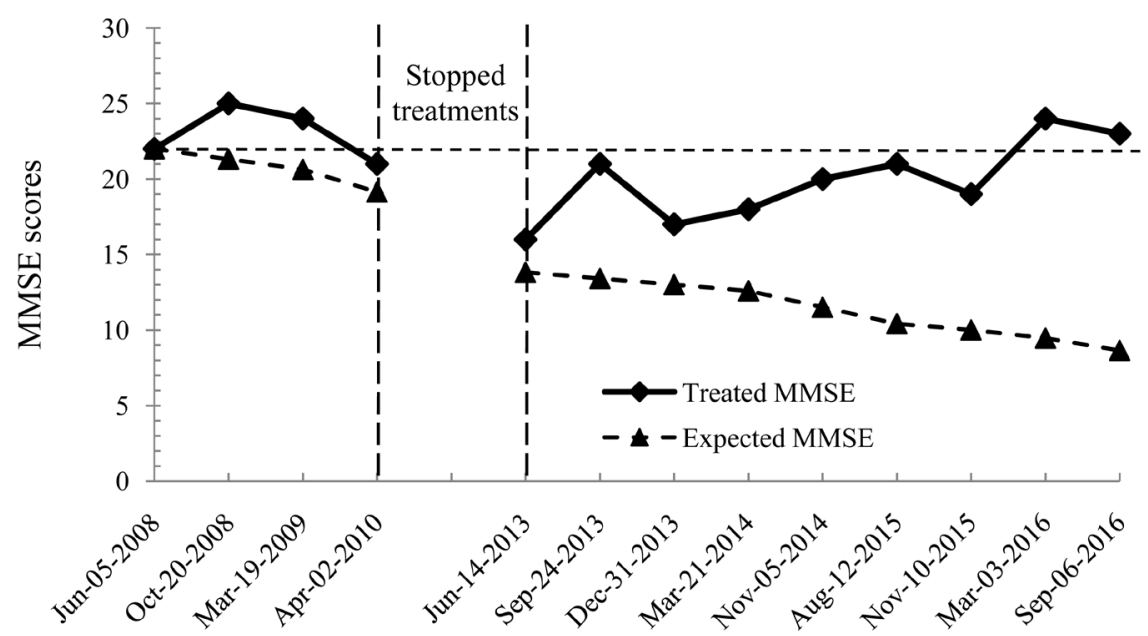

Figure 2. The MMSE changes from first visit to the latest follow up in eight year time. Note: There is a dramatic difference between observed MMSE changes and her expected decline according to a baseline-dependent mathematical MMSE prediction model published by Mendiondo et al. [3].

she continued the combined therapy this time and experienced a steady improvement in cognition. Her MMSE score increased steadily from 17 to 23. Her latest neuropsychological assessment revealed that her global cognition and daily living activities were both in normal rage, albeit a slight impairment in episodic memory. Her ability to perform complicated task, like knitting, was slightly compromised.

\section{Discussion}

With carefully evaluation, she was diagnosed as mild dementia due to Alzheimer's disease. The diagnosis was given by the following criteria, First, the patient was brought to our memory clinic for her insidious and progressive cognitive decline in half year without fluctuating characteristics. Second, she has more than two domains of cognitive impairment measured by global cognitive disorder with MMSE adjusted by education, $\leq 22$ for primary school, and the cognitive impairment has interfered with her daily activities [3] [4]. Third, her brain MRI scan showed severe atrophy of the bilateral hippocampal volume, with an age-adjusted MTA-scale abnormal score 3 or more for 75 years and more. Overall, this is quite accord with AD diagnostic criteria [5] [6] [7]. Other diseases that may cause cognitive impairment were excluded by the patient's negative results of laboratory tests.

After diagnosis of Alzheimer's disease, she received the following medicines. First, donepezil hydrochloride for improve the level of cholinergic transmitter in her brain [8]; Second, our herbal formula was prescribed for the purpose of effectively improvement of her neuron synaptic dysfunction induced by $\beta$-amyloid accumulation [9] [10] [11]. During the first stage of combinatory therapy, her cognitive function was obviously improved for about 10 months, and then retreated below the baseline at 21 months, which was already extended beyond the well-known parabola effect of donepezil. A surprising aspect of this patient is the 
dramatic drop in cognition after her quit from the treatment for about 40 months, a strong indication of the progressive Alzheimer's disease. After that, the patient was re-prescribed with the similar combined medications. Interestingly, her MMSE score improved significantly from 16 to 21 as expected, and then fell back near the baseline after six months of treatment. Intriguingly, her cognitive condition was steady improved even after the wane of beneficial effect of donepezil, and reached a nearly normal cognitive status (MMSE 23).

The cognitive outcomes of the patient after receiving eight year's sequential therapy was significantly better than the expected changes [3]. The use of donepezil in this case also showed similar pinnacle effects around three months [12] followed by a retreat to the baseline. However, with the support from our herbal formula, the beneficial effects sustained for a longer time and her cognition was steadily improved significantly thereafter. Therefore, this phenomena cannot be explained solely by the effects of donepezil, indicating that our herbal formula might work additively and independently to the cholinesterase inhibitor donepezil. Our previous work using a similar herbal formula called GAPT can markedly enhance learning and memory of $\mathrm{AD}$ rat models induced by $\mathrm{A} \beta$ injection or transgenic mice of APPV717I and APPs we/PS1dE9 [13], and also showed a significant improvement on cognitive function in patients with amnestic mild cognitive impairment, an early stage of $\mathrm{AD}(n=101)$, consistently across different cognitive cales in a small sample of clinical trial [14]. The good effects of multiple combined therapy in treating MCI (mild cognitive impairment) and SCI (subjective cognitive impairment) and $\mathrm{AD}$, was published by Dale E. Bredesen et al. [15] [16]. A therapeutic approach characterized by programmatic, personalization, as well as dubbed metabolic enhancement for neurodegeneration (MEND protocol) was used. Authors reported that those patients had unprecedented symptom improvement. However, the time of those patients treated with this MEND protocol only ranges from five to 24 months. Our case was treated and observed for much longer time than this study. Meanwhile, a two year polite study to check effects of the sequential therapy was conducted in more than two hundred patients recently. Primary results favor the sequential therapy than pure conventional medicine, which will be published soon.

\section{Conclusion}

During the treatment of this $\mathrm{AD}$ case in eight years, sequential therapy showed great potential in stabilizing and improving cognition and overall status. This indicates that the complex pathogenic process of $\mathrm{AD}$ should be treated with multiple targets medicine or a combination of multiple therapies. Well designed preclinical and clinical studies are needed to investigate the efficacy of sequential therapy for $\mathrm{AD}$ and other type of dementia.

\section{Acknowledgements}

The authors thank the outpatient and her caregivers, without whom this observation would not have been accomplished. 


\section{Funding}

This study was funded by Project on Absorption of Intellects by Institutions of Higher Education for Academic Disciplinary Innovations (the "111 Project") (No. B08006), and The Technological Platform of Clinical Evaluation and Research for New Herbal Medicinal Products (2011ZX09302-006-01), and National Natural Science Foundation of China (No. 81473518, 81573824 and 81503625).

\section{Authors' Contributions}

MQW did all the neuropsychological assessments, XKZ did the manuscript drafting, JS did the design of the investigation process, NJN and TL did the follow up of the patient, TL and YYS did the correct of the draft, LPZ did the neuroradiological assessment, PWW did the genetic analysis, SHK, YML and CML did the visits arrangement and recording, JZT did the patient consultation, provide the treatment protocol and designed the investigation process.

\section{Competing Interests}

All authors state that there is no conflict of interest.

\section{Consent for Publication}

The patient's daughter signed consent to publish.

\section{References}

[1] Cummings, J.L. (2009) Defining and Labeling Disease-Modifying Treatments for Alzheimer's Disease. Alzheimer's Dement, 5, 406-418.

[2] Wilcock, G.K. (2006) Disease-Modifying Treatments for Alzheimer's Disease: A Perspective Based on Experience with R-Flurbiprofen. Alzheimer's Dement, 2, 150 152.

[3] Mendiondo, M.S., Ashford, J.W., Kryscio, R.J. and Schmitt, F.A. (2000) Modelling Mini Mental State Examination Changes in Alzheimer's Disease. Statistics in Medicine, 19, 1607-1616.

[4] Ni, J., Shi, J., Wei, M., et al. (2015) Screening Mild Cognitive Impairment by Delayed Story Recall and Instrumental Activities of Daily Living. International Journal of Geriatric Psychiatry, 30, 888-890. https://doi.org/10.1002/gps.4317

[5] Dubois, B., Feldman, H.H., Jacova, C., et al. (2013) Research Criteria for the Diagnosis of Alzheimer's Disease: Revising the NINCDS-ADRDA Criteria. The Lancet Neurology, 6, 734-746.

[6] McKhann, G.M., Knopman, D.S., Chertkow, H., et al. (2011) The Diagnosis of Dementia Due to Alzheimer's Disease: Recommendations from the National Institute on Aging-Alzheimer's Association Workgroups on Diagnostic Guidelines for Alzheimer's Disease. Alzheimer's Dement, 7, 263-269.

[7] Tian, J., Shi, J.W.M., Zhang, X.K., Ni, J.N. and Wang, Y.Y. (2012) Chinese Characteristics of Clinical Diagnostic Criteria for Alzheimer's Disease. Chinese Medicine Frontier, 4, 2084-2087.

[8] Rogers, S.L., Farlow, M.R., Doody, R.S., Mohs, R. and Friedhoff, L.T. (1998) A 24-Week, Double-Blind, Placebo-Controlled Trial of Donepezil in Patients with Alzheimer's Disease. Donepezil Study Group. Neurology, 50, 136-145. 
https://doi.org/10.1212/WNL.50.1.136

[9] Shi, J., Tian, J., Zhang, X., et al. (2013) A Combination Extract of Renshen (Panax Ginseng), Yinyanghuo (Herba Epimedii Brevicornus), Yuanzhi (Radix Palygalae) and Jianghuang (Rhizoma Curcumae Longae) Decreases Glycogen Synthase Kinase 3beta Expression in Brain Cortex of APPV7171 Transgenic Mice. Journal of Traditional Chinese Medicine, 33, 211-217.

[10] Shi, J., Zhang, X., Yin, L., et al. (2016) Herbal Formula GAPT Prevents Beta Amyloid Deposition Induced $\mathrm{Ca}(2+) / C a l m o d u l i n-D e p e n d e n t$ Protein Kinase II and $\mathrm{Ca}(2+)$ / Calmodulin-Dependent Protein Phosphatase 2B Imbalance in APPV717I Mice. BMC Complementary and Alternative Medicine, 16, 159.

[11] Tian, J., Shi, J., Zhang, L., et al. (2009) GEPT Extract Reduces A Beta Deposition by Regulating the Balance between Production and Degradation of A Beta in APPV717I Transgenic Mice. Current Alzheimer Research, 6, 118-131. https://doi.org/10.2174/156720509787602942

[12] Winblad, B., Engedal, K., Soininen, H., et al. (2001) A 1-Year, Randomized, Placebo-Controlled Study of Donepezil in Patients with Mild to Moderate AD. Neurology, 57, 489-495. https://doi.org/10.1212/WNL.57.3.489

[13] Miao, Y.C., Tian, J., Shi, J. and Mao, M. (2012) Effects of Chinese Medicine for Tonifying the Kidney and Resolving Phlegm and Blood Stasis in Treating Patients with Amnestic Mild Cognitive Impairment: A Randomized, Double-Blind and Parallel-Controlled Trial. Journal of Chinese Integrative Medicine, 10, 390-397. https://doi.org/10.3736/jcim20120407

[14] Tian, J., Shi, J., Zhang, X. and Wang, Y. (2010) Herbal Therapy: A New Pathway for the Treatment of Alzheimer's Disease. Alzheimer's Research \& Therapy, 2, 30.

[15] Bredesen, D.E. (2014) Reversal of Cognitive Decline: A Novel Therapeutic Program. Aging, 6, 707-717. https://doi.org/10.18632/aging.100690

[16] Bredesen, D.E., Amos, E.C., Canick, J., et al. (2016) Reversal of Cognitive Decline in Alzheimer's Disease. Aging, 8, 1250-1258. https://doi.org/10.18632/aging.100981

\section{Submit or recommend next manuscript to SCIRP and we will provide best service for you:}

Accepting pre-submission inquiries through Email, Facebook, LinkedIn, Twitter, etc. A wide selection of journals (inclusive of 9 subjects, more than 200 journals)

Providing 24-hour high-quality service

User-friendly online submission system

Fair and swift peer-review system

Efficient typesetting and proofreading procedure

Display of the result of downloads and visits, as well as the number of cited articles

Maximum dissemination of your research work

Submit your manuscript at: http://papersubmission.scirp.org/

Or contactwjns@scirp.org 